Received $25^{\text {th }}$ August 2020, Accepted $24^{\text {th }}$ September 2020

Link to DOI:

10.25220/WNJ.V04.S2.0003

Journal Website: www.worldnutrijournal.org

\section{Immune development of children born from Caesarean section}

\author{
Zakiudin Munasir ${ }^{1}$, Levina Chandra Khoe ${ }^{2}$ \\ 1. Department of Pediatric, Medical Faculty, Universitas Indonesia-Cipto Mangunkusumo National \\ Hospital, Indonesia \\ 2. Department of Community Medicine, Medical Faculty, Universitas Indonesia
}

\begin{abstract}
Several studies results have shown that mode of delivery affects the health of children, and recent studies showed that elective Caesarean section (CS) is associated with aberrant short-term immune responses in the newborn infant and an increased risk of developing immune disorders. This article focused on the effect and role of the C-section on the immune development in children. Begin at pregnancy, the infant's immune system is activated and develops years after birth. In this article we find that cesarean delivery mode will influence the offspring's immune system by disrupting the intestinal tract's bacterial colonization, different levels of birth adaptive stress, and altering gene expression epigenetic regulation. Some studies have found that gut microbiome composition plays a significant role in the development of immune system along with other factors such as diet/lifestyle, antibiotic use, formula feeding, vaccination with life vaccine, and pathogen exposure. In early life, disrupted colonization induced dysbiosis that was associated with lower Bifidobacteria and higher counts of $C$. difficile. These findings are related to infant immune disease and allergy. Dysbiosis following $\mathrm{C}$-section has a huge effect of developing altered immune system, and this microbiome imbalance can be controlled by nutritional support such as maternal breast milk or the use of different combinations of prebiotics and probiotics (synbiotic) which could be beneficial for the immune and metabolic system.
\end{abstract}

Keywords: Caesarean section, gut dysbiosis, allergy, immunity, synbiotic

\section{Introduction}

Caesarean section (C-section) is a surgical procedure developed to prevent or treat lifethreatening maternal or fetal complications such as antepartum hemorrhage, fetal distress, abnormal fetal presentation, and hypertensive disease. ${ }^{1}$ The number of $\mathrm{C}$-section in the US has risen by up to $48 \%$ since 1996 . $^{2}$ The WHO recommends that in up

\footnotetext{
Corresponding author:

Prof. Dr. dr. Zakiudin Munasir, SpA(K)

Cipto Mangunkusumo National Hospital

Jakarta, Indonesia

Email: zakiudin.munasir@gmail.com
}

to $15 \%$ of deliveries, $\mathrm{C}$-section may be indicated. However, 37 of 60 developed countries currently surpassing this guideline and taking women undergoing pre-labor, elective $\mathrm{C}$-section without a clear medical indication may present risks to the child. ${ }^{2,3}$ Simultaneously with the increase of rising C-section levels, there has been an increasing number of autoimmune and allergic diseases. ${ }^{4}$ Possible mechanisms for these associations may include lower and untimely activation of the fetal immune system due to lack of stress response caused by contraction of the uterus and fetal hypoxia, ${ }^{3}$ and alteration of the bacterial colonization of the infant's gut after C-section. ${ }^{2}$

Dysbiosis occurs when there is irregular colonization of the body intestine, also considered to be a mismatch between commensal and pathogenic 
microbes. ${ }^{5}$ Intestinal bacterial flora is critical for immune growth, and children with atopic diseases have been shown to have lower gut levels of Bacteroides and Bifidobacterium species than children without atopic disease. Bifidobacteria are associated with reduced risk of atopic disease in children. C-section has been shown to disrupt and change the development of bacterial intestinal flora in infants. This may, in turn, alter development of the immune system and increase the risk of atopic disease in children. ${ }^{6}$

Uterus contraction and fetal hypoxia during childbirth cause a stress response in both the mother and the fetus, raising the substantial levels of cortisol and catecholamine in newborns. Multiple studies show a low level of cortisol and catecholamine in the absence of contraction in the C-section delivery, variations in levels of stress hormones at birth may alter the immune responses and influence the immune system later in life. ${ }^{3,7}$ Cortisol may remove uterine preleukemic clones. ${ }^{7}$ There is also evidence of altered DNA methylation patterns that are important to immune regulation after $\mathrm{C}$-section. ${ }^{7,8}$

\section{Methods}

This article focused on how the delivery mode affects the neonate's immune system. This analysis was prepared by conducting a search to identify related journals and articles published in 2010 until before April 2020 in several electronic databases, i.e. Pubmed and Medline. The search terms included variants of "delivery mode," "cesarean section," "immune system," "allergy," "asthma," "diabetes," "celiac disease," "allergy to food," "prebiotic" and "probiotic" Additional articles were found by manual search, either in English or Indonesian, from manual references cited in relevant reviews.

\section{Results and Discussion}

\section{Immune development in early life}

Immune activation starts from insemination as the first immune system exposure to paternal alloantigens. Likewise, it contains paternal immune cells and substances, such as estrogen and testosterone, prostaglandins and various signaling molecules, including IL-8, TGF-b, and IFN-C. It also promotes the transfer and expansion of regulatory T cells (Treg) and promotes the activation and proliferation of dendritic cells (DCs) in responsive cells. Treg is probably involved in $\mathrm{T}$ cells suppression of the mother's effector that promote the success of pregnancy. B cells secrete antibodies that once shield paternal antigens found in the trophoblast on the fetal-maternal interface. The phenotypes of immune cell function, i.e. DC, turning or remaining unchanged and thus tolerant, are modulated by a wide range of molecules formed or secreted by the trophoblast itself, such as the human chorionic gonadotropin (hCG), Heme Oxygenase-1 (HO-1), Regulated on activation, normal $T$ cell expressed and secreted (RANTES), and pregnancy-specific glycoprotein (PSG). ${ }^{9}$ Additionally, cell-secreted molecules such as Gal-1 secreted by uterine Natural Killer cells (uNKs) and uterine Mast Cells (uMCs) can have a positive effect on trophoblast physiology while making maternal $\mathrm{T}$ cells become receptive or remain to the fetus. These findings suggest that seminal plasma plays an important role in the uterine system preparation for the embryo implantation. ${ }^{9,10}$

A labor will stimulate immune response within the uterine cavity which will be absent in the case of elective C-sections. The production of proinflammatory cytokines in the uterine environment such as interleukin (IL)-1 $\beta$, IL-6, IL-8 and tumour necrosis factor- $\alpha$ (TNF- $\alpha)$ is thought to be responsible for an activation of the fetal immune system during labor. This reflects the increased of leukocytes in neonates' circulation. ${ }^{11}$

As in all mammals, fetal immune production has been observed at 3-4 weeks of gestation, where the pluripotent erythroid and granulomacrophage progenitors can be found in human embryo's yolk sac. These primitive cells migrate to the liver, which becomes the main haemopoiesis site at $5 \pm 6$ weeks of gestation, thereby increasing the size of the liver and increasing the number of nucleated cells. A discrete granulocyte/macrophage population is growing at this time. ${ }^{12}$ Nevertheless, the neutrophil will not be noticed until the end of the third trimester. As a result, newborn and, in particular, premature infants have impaired neutrophil activity, putting the child at risk of bacterial infections. ${ }^{13}$

In the uterus, the fetus has an immune system that is tolerant to maternal alloantigens. The 
mother's uterine natural killer (uNKs) cells, immature dendritic cells (iDCs), $\mathrm{T}$ cells and macrophages help to modulate the uterine environment in order to support successful pregnancy. ${ }^{14}$ This system will continue after birth when the enormous exposure to environmental antigens, many of which are derived from intestinal commensal bacteria, calls for a rapid change in order establish distinct immune responses that are appropriate for the infant in early life. ${ }^{13}$

The immune system is not fully functioning at birth and therefore infants are at an elevated risk of infection. After birth, infants are moving from the sterile environment of the womb to the wider world, exposed to a wide range of diseases they have never experienced and have no protection against. The 'hygiene hypothesis' theory postulates that early life preparation of the immune system arising from essential immune modulatory exposures helps to stimulate regulatory mechanisms that protect against infectious diseases and allergy. ${ }^{15}$

The definition of allergy has also evolved over the years, and currently, it is considered an umbrella term for an immune defect resulting in the lack of tolerance to usually harmless antigens. ${ }^{16}$ Tolerance is antigen-specific and the loss of tolerance in allergies seems to be related to the timing, rate and context of environmental exposures in early life, including bacterial colonization of the infant gut. ${ }^{17}$ Specific early microbial intestinal exposure is thought to significantly reduce the incidence of inflammatory, autoimmune and atopic diseases further fuel the scientific view that microbial colonization plays an important role in regulating and fine-tuning the immune system throughout life. ${ }^{12,18}$ As a major part of the mucosal immune system, the gut-associated lymphoid tissue (GALT) has $M$ cells that collect small particles and effectively transport them from the intestine to organized lymphoid tissue and cause mucosal immune reaction. ${ }^{18}$

\section{The innate and adaptive immune system}

Microorganisms that successfully enter the body will be immediately recognized by the cells and mechanisms of the innate immune system within hours of an antigen-specific response being developed by the adaptive immune system a few days later. ${ }^{18,19}$. Innate immune cells are commonly located along with physical barriers such as skin and mucous membranes, where they release defensive biomolecules such as defensins and complements. Upon pathogen identification, dendritic cells and macrophages (often known as antigen-presenting cells, APCs) are activated to phagocytose the invader, digest, and process pieces (antigens) on the cell surface in the form of major histocompatibility complex (MHC) molecules. ${ }^{20}$ Adaptive cells include T-cells that primarily target intracellular microbes, along with B-cells that produce antibodies, most of which target extracellular pathogens. Innate immunity is primarily involved in the initial control of microbes. Adaptive defense is mainly concerned with the final clearance of the invader. Adaptive immunity also maintains certain cells as immune memory cells; these antigen-experienced memory cells do not need the same degree of innate immune support for secondary infection with the same (or similar) pathogen and react much faster and more efficiently. ${ }^{19}$

\section{Impact of C-section on child's immune development}

For the postnatal development of the immune system, it is important to microbially colonize the infant gut gastrointestinal tract. C-Section delivered infants, either lacked or displayed a slow colonization of one of the main intestinal phylums, the Bacteroidetes, because the infant does not enter the mother's birth canal. Therefore, the delayed colonization leads to low-diversity of the intestinal microbiota, which leads to a lack of regulatory $\mathrm{T}$ cell and/or Th1-like responses to avoid Th2 domination. In addition, the dysbiosis in the C-section children is associated with lower circulation of CXCL10 and CXCL11 chemokines related to Th-1 when the risk of allergies increases. ${ }^{6}$

The "hygiene hypothesis" was introduced in 1989 when researchers argued that the increase in allergic diseases was due to a lack in microbial exposure due to improved sanitization practices following a low incidence of early childhood allergy infection. ${ }^{21}$ Modern medicines and changes in sanitation and public health have led to an era of unprecedented cleanliness and the near eradication 
of previously common pathogens. However, these improvements coincided with the rise of autoimmune diseases and other immune disorders. Strachan and others indicated that a certain amount of microbial stimulation is required to prevent disease. ${ }^{5}$ Microbiome includes both pathogens and commensals, which play a role in immune development.

In addition to exposure to parasites outside the body, as many as 100 trillion microbes colonize barrier sites within our body, most of which are in the intestine, indicating how the gut serves as one of the major organ in which the immune system is developed. The composition of the microbiome is dynamic and strongly affected by external factors such as mode of birth, diet/lifestyle, antibiotic usage, vaccination, formula feeding and pathogen exposure. This process is necessary to educate the immune system on how to respond to a wide range of stimuli encountered. Given the intricate communication between the gut microbiota and immune system, which plays a role in the development of tolerance and immune programming, disruption of the diversity and/or the function of the microbiota referred to as dysbiosis may increase risk of immune disorders such as allergy. $^{21}$

There are significant shifts in the makeup of the intestinal microbiota during early life. The intestines are sterile at birth. Within a few hours, the bacteria will begin to appear in the feces. The bacteria colonizing the infant intestine during the first days of childhood derive mainly from the mother and the environment. The mode of transmission is therefore the key determinant of intestinal microbiota. ${ }^{22}$ Vaginal infant microbiota $(<24 \mathrm{~h}$ postpartum $)$ resembles the vaginal microbiota of the mother and is common across various body habitats (skin, oral, nasopharynx, birth canal, and feces), whereas in neonates born by $\mathrm{C}$-section is identical to the skin microbiota of the mother. ${ }^{6,22,25}$ The $\mathrm{C}$-section was correlated with lower Bifidobacteria counts, lower colonization rates and counts of $B$. fragilis species, and higher counts of $C$. difficile. ${ }^{22}$ Infants with a higher number of Bifidobacteria species at 1 month of age have higher rates of salivary IgA, which also correlates with allergic symptoms defense. ${ }^{5}$ The additional prophylactic antibiotic given in $\mathrm{C}$-section may also lead to aberrant microbial colonization in the infant intestine and may increase susceptibility to metabolic disease in later life. ${ }^{26} \mathrm{C}$. difficile was also the single gut microbe investigated that was associated with an increased risk for asthma, but this association was restricted to those children with atopic parents. ${ }^{25}$

Another research stated that there is a lack of stress hormones in the infant born by the C-section. Contraction and oxygen hypoxia during vaginal delivery are known to promote the development of stress hormones such as catecholamine and cortisol. ${ }^{3,27}$ Cortisol elevation at birth is a predictor of hypothalamic-pituitary-adrenal axis activation of the immune system, lung and organ maturation, and neurogenesis. Vaginal delivery and its effects on elevating glucocorticoids have been associated with increased maturation of the organs, including the intestine. These two stress hormones, on the other hand, are less present in infants born by $\mathrm{C}$-section before labor. ${ }^{3}$ Another experimental study showed that the use of synthetic oxytocin, antibiotics or infants delivered by the $\mathrm{C}$-section had higher global DNA methylation in the cord blood cells, and this neonatal epigenetic modification has implications for the wellbeing of the offspring. ${ }^{28}$ Higher global DNA methylation could be involved in silencing the pathway that controls the balance between T-helper type 1 and 2 cells, which could lead to a higher risk of developing immune diseases. ${ }^{29}$ Further studies on this hypothesis are needed.

\section{Health risks associated with altered immune development on child born by $C$-section}

Recent studies have shown that C-section delivery has been associated with short-term aberrant immune responses in newborn infants and a higher risk of developing immune diseases such as asthma, allergies, neonatal respiratory disease, type 1 diabetes, celiac disease and malignancy. ${ }^{3,26,30}$ As mentioned, C-section delivery may alter immune development by three means: (1) disrupting bacterial colonization of the intestines; (2) mounting poor and inadequate stress response; and (3) altering epigenetic regulation of gene expression through DNA methylation. ${ }^{3}$ 


\section{Respiratory system}

The risk of respiratory morbidity after elective CS is mainly related to gestational age in terms of neonates, but it also shows that the lack of work in elective CS may explain reduced production of catecholamines (also in preterm infants). ${ }^{3,27}$ This lack of hormones altered the lung adaptation and the associated clinical symptoms such as benign intermittent tachypnea, chronic neonate pulmonary hypertension, pulmonary air leakage and hypoxemia. ${ }^{27}$

\section{Asthma, allergic rhinitis and food allergy}

There are reports that there is a risk of developing allergic disease, allergic rhinitis, asthma and asthma hospitalization and food allergy, whereas there was no association with inhalant atopy and eczema/atopic dermatitis. ${ }^{25,31}$ Almqvist et $\mathrm{al}^{32}$ reported the outcome of a cohort study in 2012 in which there was an increased risk of asthma and asthma diagnosis during the follow-up year in children born with a C-section. Increased risk of asthma medication in the emergency-born, but not elective, category suggests that there is no causal effect due to vaginal microflora. A more likely explanation should be sought in the emergency Csection. Changed immune function in infants delivered by $\mathrm{C}$-section compared to vaginal delivery is documented by reduced production of cytokines involved in neonatal immunity, in this respect increased levels of IL-13 and IFN-supposed associated with atopy. ${ }^{27}$ The frequent presence of C. difficile in the feces at 1 month post-partum by $\mathrm{C}$ section delivery has an association with the development of wheeze, eczema, and sensitization to food allergens in the first 2 years of life. ${ }^{25}$

A recent cohort study from Mitselou $\mathrm{N}$ et $a l^{33}$ using a large population database, shown an increased risk of food allergy in children born through elective or emergency Caesarean delivery compared to those delivered vaginally. This positive association aligned with the theory that exposure to vaginal microflora might reduce the risk of offspring atopic manifestations. The altered composition of the intestinal flora involves these improvements to the immune response to a more atopic profile. The colonization of intestinal microbes appears to stimulate a non-allergic Th1 reaction of the immune system while $\operatorname{IgE}$ synthesis seems to be downregulating. C-Sectional children are seemed to have weak immune recognition, especially when born to an allergic mother to foods that are supposed to be tolerated..$^{34}$

\section{Type-1 diabetes mellitus}

Recent studies have shown that children born in the C-section are at more than $20 \%$ higher risk of childhood-onset type 1 diabetes mellitus relative to those born per-vaginam. ${ }^{3}$ Other studies have shown that increased type 1 diabetes mellitus is associated with altered development of the immune system. ${ }^{27,35,36}$ In addition, it has been previously suggested that children with decreased early-life exposure to microorganisms may be at increased risk for type 1 diabetes. ${ }^{27}$

\section{Gastrointestinal disease}

Bacterial flora of the newborns plays a role in the development of celiac disease. Studies showed a positive association between elective $\mathrm{C}$-section and development of celiac disease, but not for an emergency C-section. ${ }^{35,37}$ However, the incidence of inflammatory bowel disease has not been affected by the mode of delivery. ${ }^{3}$ Maternal microbial and immune status, infant nutrition and antibiotic exposure affect the development of the intestinal microbiome and therefore the development of the immune system. Dysbiosis can lead to immunemediated diseases, including allergies and necrotizing enterocolitis (NEC). ${ }^{5}$

\section{Malignancy}

There are indications of an increased risk of cancer (leukemia, ${ }^{3,7}$ neuroblastoma, testicular cancer) in children born by elective C-section compared to vaginal delivery. Although these correlations have not been verified or refuted in other studies. ${ }^{3}$

\section{Impact of C-section on risk of developing allergy}

In this study, mode of delivery was correlated with early childhood asthma and allergy. Children born in the $\mathrm{C}$-section were at an increased risk of developing 
childhood asthma, food allergy perhaps associated with risk for allergic rhinitis and atopic dermatitis. Although the mechanisms are not fully understood, it could involve the confounder such as maternal smoking, socioeconomic status or family history of asthma. ${ }^{32}$ Microflora plays a number of critical roles in the intestinal colonization and production and homeostasis of the immune system. Some of the strongest data linking dysbiosis and allergies are epidemiological associations with conditions that cause dysbiosis - particularly C-section. ${ }^{38}$

The predominant sources of microbes for the initial colonization of GIT after birth are maternal microbiota, especially during birth delivery, and infant diet (breast versus formula feeding). ${ }^{22}$ The mode of delivery is a key factor that shapes the developing infant microbiota and, in this regard, infants delivered by the $\mathrm{C}$-section have been reported to have an enteric microbiota that differs from infants delivered by the vagina, both in time of colonization and in composition. The modified microbiota may have an immunomodulatory effect that increases the child's susceptibility to poor immune recognition of foods that should be tolerated by food-specific $\operatorname{IgE}$ found in infants delivered by $\mathrm{C}$-section. ${ }^{34}$

\section{Nutrition intervention to reduce risk of developing allergy}

Nutritional measures are key to early-life immune functioning by impacting the gut barrier through strengthening the intestinal microbiota and immune tolerance. ${ }^{39}$ Breast milk promotes the colonization and maturation of the infant gut microbiome, as it contains beneficial bacteria such as Staphylococcus, Streptococcus, Serratia, Pseudomonas, Corynebacterium, Ralstonia, Propionibacterium, Sphingomonas and Bradyrhizobiaceae. Breast milk definitely contributes to stimulate the growth of the microbiota strain specific to the gut Bifidobacterium found in the intestines of nursing infants along with Bacteroides and it can have an important effect on the development of immune tolerance and genomic capacity for metabolizing the human milk oligosaccharides (HMOs)..$^{24,40}$ As a prebiotic, HMOs have effects and benefits in the alteration of the intestinal microbiota, pathogens' counteradhesive effects, epithelial cell response modulation and immune system growth. HMOs are therefore beneficial in the treatment of dysbiosis in breastfeeding children. Moreover, some studies have shown that the role of nutrition intervention could also play in gut microbiota production for unbreastfed babies. ${ }^{41,42}$

Some clinical and preclinical studies demonstrate that synbiotics can prevent certain allergic diseases. This contributed to the use of synbiotic to compensate for early microbial bowel disruptions. ${ }^{43}$ World Allergy Organization (WAO) recommended using probiotics to avoid food allergy in pregnant women at high risk of having an allergic infant, women who breastfeed infants with high risk of developing allergy and infants with high risk of developing allergy. ${ }^{44}$ Prebiotic supplementation also recommended by WAO panel to prevent allergy in not-exclusively breastfed infants regardless of their allergic risk. However for the prebiotic recommendation is conditional and need a further strong certainty of evidences. ${ }^{45}$

In the JULIUS study conducted by Chua et al, ${ }^{46}$ the effect synbiotics on the gut microbiota has been determined in infants born by $\mathrm{C}$-section. The result has shown that supplementation with specific synbiotic mixture (a mixture of short-chain galactooligosaccharides (scGOS) and long-chain fructooligosaccharides (lcFOS) and B.Breve M16-V in Csection-born infants allows rapid colonization of Bifidobacteria from the first days of life and offers promising nutritional intervention in dysbiosis. Furthermore, safety follow-up showed a significantly lower incidence of reported skinrelated disorders and atopic dermatitis among those treated with this synbiotic compared to the control group.

A randomized study of 90 exclusively atopic dermatitis (AD) at large-formula (eHF) plus synbiotic (a mixture of scGOS and LcFOS and Bifidobacterium breve M-16 V) showed significant modulation of the intestinal microbiota, with high percentages of bifidobacteria and lower percentages of Clostridium litusebrense/Clostridium histolyticum and Eubacterium rectale/Eubacterium. The synbiotic impact was sustained for one year following and the incidence of symptoms such as asthma and the use of asthma medications was significantly reduced. ${ }^{47}$ Potential role of synthesized human milk oligosaccharides (HMOs) has been 
studied recently and are considered safe for infant nutrition to support microflora development. ${ }^{48}$

In addition to this, future approach that provide combination of synbiotic and hydrolyzed formula might be modulate immune system in infants to support tolerance development. Hydrolyzed formula still contains certain protein fractions of different sizes. Some of them may have a role in inducing oral tolerance as recently shown in a study in combination of prebiotic mixture of scGOS and lcFOS lowering sensitization against cow's milkspecific IgG1 and higher level of dendritic and Treg cell, demonstrating its immune tolerance-inducing capacity. ${ }^{49}$ This indicates that the use of synbiotic in early life may be beneficial to compensate for gut microbiota dysbiosis, but more study is needed on the benefits of patient outcomes, particularly in terms of allergic outcomes.

\section{Conclusion}

Birth process can be a critical time point that decides the immune function of the offspring in later life. The mode of delivery has been associated with several infant conditions. Studies have shown that $\mathrm{C}$-section has been associated with immune and allergic diseases caused by altered immune development compared to vaginal delivery. The potential mechanism for the $\mathrm{C}$-section affecting the development of the immune system may be the alteration of bacterial colonization that causes dysbiosis or may be associated with adverse birth stress reactions and epigenetic modification of gene expression in the immune system. Moreover, dysbiosis causes immune imbalances and is thought to cause allergy, and synbiotic use may improve the immune condition of children.

\section{Conflict of Interest}

Authors declared no conflict of interest regarding this article.

\section{Open Access}

This article is distributed under the terms of the Creative Commons Attribution 4.0 International Licence (http://creativecommons.org/licenses/by/4.0/), which permits unrestricted use, distribution, and reproduction in any medium, provided you give appropriate credit to the original author(s) and the source, provide a link to the Creative Commons license, and indicate if changes were made.

\section{Reference}

1. Boerma T, Ronsmans C, Melesse DY, Barros AJ, Barros FC, Juan L, et al. Global epidemiology of use of and disparities in caesarean sections. Lancet. 2018;392(10155):1341-8.

2. Neu J, Rushing J. Cesarean versus vaginal delivery: long-term infant outcomes and the hygiene hypothesis. Clin Perinatol. 2011;38(2):321-31.

3. Cho CE, Norman M. Cesarean section and development of the immune system in the offspring. Am J Obstet Gynecol. 2013;208(4):249-54.

4. Huang L, Chen Q, Zhao Y, Wang W, Fang F, Bao Y. Is elective cesarean section associated with a higher risk of asthma? A meta-analysis. $J$ Asthma. 2015;52(1):16-25.

5. Houghteling PD, Walker WA. From birth to 'immuno-health', allergies and enterocolitis. J Clin Gastroenterol. 2015;49(0 1):S7.

6. Jakobsson HE, Abrahamsson TR, Jenmalm MC, Harris K, Quince C, Jernberg C, et al. Decreased gut microbiota diversity, delayed Bacteroidetes colonisation and reduced Th1 responses in infants delivered by caesarean section. Gut. 2014;63(4):55966.

7. Wang R, Wiemels JL, Metayer C, Morimoto L, Francis SS, Kadan-Lottick N, et al. Cesarean section and risk of childhood acute lymphoblastic leukemia in a population-based, record-linkage study in California. Am J Epidemiol. 2017;185(2):96-105.

8. Franz MB, Poterauer M, Elhenicky M, Stary S, Birner P, Vinatzer U, et al. Global and single gene DNA methylation in umbilical cord blood cells after elective caesarean: a pilot study. Eur J Obstet Gynecol Reprod Biol. 2014;179:121-4.

9. Munoz-Suano A, Hamilton AB, Betz AG. Gimme shelter: the immune system during pregnancy. Immunol Rev. 2011;241(1):20-38.

10. Zenclussen AC. Adaptive immune responses during pregnancy. Am J Reprod Immunol. 2013;69(4):291303.

11. Francino MP. Birth mode-related differences in gut microbiota colonization and immune system development. Ann Nutr Metab. 2018;73(3):12-6.

12. Bhattacharya N, Stubblefield PG. Human Fetal Growth and Development: First and Second Trimesters: Springer; 2016. 
13. Simon AK, Hollander GA, McMichael A. Evolution of the immune system in humans from infancy to old age. $P$ Roy Soc B-Bio Sci. 2015;282(1821):20143085.

14. PrabhuDas M, Bonney E, Caron K, Dey S, Erlebacher A, Fazleabas A, et al. Immune mechanisms at the maternal-fetal interface: perspectives and challenges. Nat Immunol. 2015;16(4):328-34.

15. Wopereis H, Oozeer R, Knipping K, Belzer C, Knol J. The first thousand days-intestinal microbiology of early life: establishing a symbiosis. Pediat Allerg Imm-UK. 2014;25(5):428-38.

16. Haahtela T, Holgate S, Pawankar R, Akdis CA, Benjaponpitak S, Caraballo L, et al. The Biodiversity Hypothesis and Allergic Disease: World Allergy Organization Position Statement. World Allergy Organ J. 2013 Jan 31;6(1):3

17. Benedetto AD, Kubo A, Beck LA. Skin Barrier Disruption: A Requirement for Allergen Sensitization? J Invest Dermatol. 2012 Mar;132(3 Pt 2):949-63

18. Martin R, Nauta A, Ben Amor K, Knippels L, Knol J, Garssen J. Early life: gut microbiota and immune development in infancy. Benef Microbes. 2010;1(4):367-82.

19. Turvey SE, Broide DH. Innate immunity. $J$ Allergy Clin Immunol. 2010;125(2 Suppl 2):S24-S32.

20. Goenka A, Kollmann TR. Development of immunity in early life. $J$ Infection. 2015;71:S112-S20.

21. Shu S-A, Yuen AWT, Woo E, Chu K-H, Kwan H-S, Yang G-X, et al. Microbiota and Food Allergy. Clin Rev Allerg Immu. 2019;57(1):83-97.

22. Houghteling PD, Walker WA. Why is initial bacterial colonization of the intestine important to the infant's and child's health? J Pediatr Gastr Nutr. 2015;60(3):294.

23. Dominguez-Bello MG, Costello EK, Contreras M, Magris M, Hidalgo G, Fierer N, et al. Delivery mode shapes the acquisition and structure of the initial microbiota across multiple body habitats in newborns. PNAS. 2010;107(26):11971-5.

24. Dominguez-Bello MG, De Jesus-Laboy KM, Shen N, Cox LM, Amir A, Gonzalez A, et al. Partial restoration of the microbiota of cesarean-born infants via vaginal microbial transfer. Nat Med. 2016;22(3):250.

25. Van Nimwegen FA, Penders J, Stobberingh EE, Postma DS, Koppelman GH, Kerkhof M, et al. Mode and place of delivery, gastrointestinal microbiota, and their influence on asthma and atopy. $J$ Allergy Clin Immunol. 2011;128(5):948-55. e3.

26. Mueller NT, Whyatt R, Hoepner L, Oberfield S, Dominguez-Bello MG, Widen E, et al. Prenatal exposure to antibiotics, cesarean section and risk of childhood obesity. Int J Obesity. 2015;39(4):665-70.

27. Boutsikou T, Malamitsi-Puchner A. Caesarean section: impact on mother and child. Acta Paediatr. 2011;100(12):1518-22.

28. Dahlen HG, Kennedy HP, Anderson CM, Bell AF, Clark A, Foureur M, et al. The EPIIC hypothesis: intrapartum effects on the neonatal epigenome and consequent health outcomes. Med Hypotheses. 2013;80(5):656-62.

29. Martino D, Prescott S. Silent mysteries: epigenetic paradigms could hold the key to conquering the epidemic of allergy and immune disease. Allergy. 2010;65(1):7-15.

30. Sevelsted A, Stokholm J, Bønnelykke K, Bisgaard H. Cesarean section and chronic immune disorders. Pediatrics. 2015;135(1):e92-e8.

31. Eichenfield LF, Tom WL, Chamlin SL, Feldman SR, Hanifin JM, Simpson EL, et al. Guidelines of care for the management of atopic dermatitis: section 1 . Diagnosis and assessment of atopic dermatitis. $\mathrm{J} \mathrm{Am}$ Acad Dermatol. 2014;70(2):338-51.

32. Almqvist $C$, Öberg AS. The association between caesarean section and asthma or allergic disease continues to challenge. Acta Paediatr. 2017;103(4):349-51

33. Mitselou N, Hallberg J, Stephansson O, Almqvist C, Melen E, Ludvigsson JF. Cesarean delivery, preterm birth, and risk of food allergy: Nationwide Swedish cohort study of more than 1 million children. $J$ Allergy Clin Immunol. 2018 Nov;142(5):1510-14.e2.

34. Molloy J, Allen K, Collier F, Tang MLK, Ward AC, Vuillermin $P$. The potential link between gut microbiota and IgE-mediated food allergy in early life. Int $J$ Environ Res Public Health. 2013;10(12):7235-56.

35. Torrazza RM, Neu J. The developing intestinal microbiome and its relationship to health and disease in the neonate. Am J Perinatol. 2011;31(1):S29-S34.

36. Vehik K, Dabelea D. Why are C-section deliveries linked to childhood type 1 diabetes? Diabetes. 2012;61(1):36-7.

37. Mårild K, Stephansson O, Montgomery S, Murray JA, Ludvigsson JF. Pregnancy outcome and risk of celiac disease in offspring: a nationwide case-control study. Gastroenterology. 2012;142(1):39-45. e3.

38. Ipci K, Altıntoprak N, Muluk NB, Senturk M, Cingi C. The possible mechanisms of the human microbiome in allergic diseases. Eur Arch OtoRhino-L. 2017;274(2):617-26.

39. Renz H, Holt PG, Inouye M, Logan AC, Prescott SL, Sly PD. An exposome perspective: Early-life events and immune development in a changing world. $J$ Allergy Clin Immunol 2017; 140:24-40. 
40. Mueller NT, Bakacs E, Combellick J, Grigoryan Z, Dominguez-Bello MG. The infant microbiome development: mom matters. Trends Mol Med. 2015;21(2):109-17.

41. Vandenplas Y, Munasir Z, Hegar B, Kumarawati D, Suryawan A, Kadim M, et al. A perspective on partially hydrolyzed protein infant formula in nonexclusively breastfed infants. Korean J Pediatr. 2019;62(5):149.

42. Botteman MF, Munasir Z, Sulistomo AW, Horodniceanu EG, Bhanegaonkar AJ, Ji X, et al. Economic value of atopic dermatitis prevention via partially-hydrolyzed whey-based infant formula (PHF-W) use in high-risk, non-exclusively breastfed, Indonesian urban infants: results of a costeffectiveness model. World Nut J. 2019;2(2):43-55.

43 Moya-Pérez A, Luczynski P, Renes IB, Wang S, Borre $\mathrm{Y}$, Anthony Ryan $\mathrm{C}$, et al. Intervention strategies for cesarean section-induced alterations in the microbiota-gut-brain axis. Nutr Rev. 2017;75(4):225-40.

44. Fiocchi A, Pawankar R, Cuello-Garcia C, Ahn K, AlHammadi S, Agarwal A, et al. World Allergy Organization-McMaster University Guidelines for Allergic Disease Prevention (GLAD-P): Probiotics

45. Cuello-Garcia CA, Fiocchi A, Pawankar R,YepesNuñez JJ, Morgano GP, Zhang Y, Ahn K, et al. World Allergy Organization-McMaster University
Guidelines for Allergic Disease Prevention (GLADP): Prebiotics

46. Chua MC, Ben-Amor K, Lay C, Goh AE, Chiang $\mathrm{WC}$, Rao R, et al. Effect of synbiotic on the gut microbiota of cesarean delivered infants: a randomized, double-blind, multicenter study. $J$ Pediatr Gastr Nutr. 2017;65(1):102-6.

47. Van der Aa L, Heymans H, Van Aalderen W, Sillevis Smitt J, Knol J, Ben Amor K, et al. Effect of a new synbiotic mixture on atopic dermatitis in infants: a randomized-controlled trial. Clin Exp Allergy. 2010;40(5):795-804.

48. Hegar B, Wibowo Y, Basrowi RW, Ranuh RG, Sudarmo SM, Munasir Z, Atthiyah AF, Widodo AD, Supriatmo , Kadim M, Suryawan A, Diana NR, Manoppo C, Vandenplas Y. The Role of Two Human Milk Oligosaccharides, 2'-Fucosyllactose and Lacto-N-Neotetraose, in Infant Nutrition. Pediatr Gastroenterol Hepatol Nutr. 2019 Jul;22(4):330-40.

49. Boyle, R., et al., Prebiotic-supplemented partially hydrolysed cow's milk formula for the prevention of eczema in high-risk infants: a randomized controlled trial. Allergy. 2016.71:701-10. 